\title{
Spray-On Socks: Ethics, Agency, and the Design of Product-Service Systems Damon Taylor
}

\section{The Socks of the Future}

On different sides of the city-in the future, two consumers get out of bed and each puts on their socks. Janet's are soft and luxurious-made from a combination of Spandex, cotton, polyester, and Kevlar. They are hard-wearing, yet warm and yielding, and they slip onto her feet in a pleasurable and satisfying manner. They are a happy yellow; and as she looks at them first thing in the morning, she smiles. The electrochromatic structure woven through each sock, which alters the color through a minute electrical charge that is passed through the weave, allows the socks to change hue and pattern throughout the day according to her needs: black for work, a cheerful red for drinks later, striped for relaxing around the house. The firmware updates that come from her sock-service provider ensure that she always has the latest patterns and colors. Enjoying the feel of the socks on her feet, she experiences pleasure. Janet loves her socks.

Across town, Faisal reaches for a can and-holding out each foot in turn, sprays onto them a compound consisting of cotton fibers suspended in a solvent, blended with a range of polymers. As he watches for a moment, the coating dries, the fibers matt down to create a felting effect, and a smooth layer forms over the surface of his feet. He finds the initial fit too restrictive, though, so he flexes his feet a little, causing the applied layer to come loose until he can fully peel off the newly formed socks. After rubbing and stretching them, he puts them back on and continues dressing. Faisal does not love his socks, but they work; this utilitarian effectiveness is enough for him. He knows that after a few washes the socks will become worn and baggy, but then he can simply feed them into the home recycling unit, supplied along with the rest of the apparatus necessary by his sock-service provider, and, with a small top-up of the raw materials, he can spray on a new pair. Thus, he gets functional socks that, because they require no weaving or stitching and use only the absolute minimum of resources, are both cheap and energy efficient. 
Neither of these scenarios is too far-fetched. The technology for each already exists. Super-durable socks made of the constituents described, ${ }^{1}$ textiles that change color upon the application of an electrical current, ${ }^{2}$ spray-on garments: ${ }^{3}$ All these elements are currently commercially available. Thus, both possibilities are perfectly realizable for the ways in which people might use socks in the near future. So, technological progress could be said to be setting new parameters for the way in which socks can and will be used, opening up new vistas for sock designers of tomorrow as innovative ways of making and using create new opportunities to be addressed.

The primary question here may look like an engineering one: how to provide the best sock-system. However, the more critical question is actually an ethical one, concerned with what sort of sock-world we want. In the past decade or so, such innovations in the way products are brought to the market and consumed-ones that combine the provision or use of material artifacts and the qualities of a service in an integrated system-have come to be called Product-Service Systems (PSS). ${ }^{4}$ The United Nations Environment Programme defines a PSS as a shift "from designing and selling physical products only, to selling a system of products and services which are jointly capable of fulfilling specific client demands." ${ }^{5}$ The business logic runs that if the value for the producer can no longer come from making an artifact and selling it for more than the production cost, then it must come in the form of an expansion and monetizing of the provision phase of the life-course of commodities. Thus, in the post-mass-industrial West, it is in the exploitation of the way in which what is produced is incorporated into the matrix of everyday life, that is to say in use, that the potential for making a profit has been located.

It may be absurd to suggest that people in the future would choose to consume sock-services as convoluted as the ones described. However, the development of PSS as a model of provision makes clear that such schemes are being considered and it is certainly not beyond the realm of imagination to suppose that producers are thinking of ways to make these kinds of arrangements a reality. Through an analysis of the interaction of the ontological coordinates set by PSS itself-the product, the service, and the system-it is argued that the key challenge that the designer faces in such situations is ethical in character. Not because designers are considered to be the ultimate arbiters of good or bad; rather, since the focus of research has moved from the nature of objects to the behavior of people, it is suggested that design for use has become the design of use. The intention, therefore, is to question what conditions govern the way in which designers can operate in the emerging systemic paradigm of design, making, and provision.

Opportunities for Sustainable Solutions

(Paris: United Nations Environment

Programme, 2002), 4. 


\section{Service Providers}

The future that Faisal and Janet inhabit may not be so far away. Since 1999 the Internet company Blacksocks ${ }^{\mathrm{TM}}$ has run what could be termed a sock provision system. ${ }^{6}$ By using the access and distribution models made possible by the expansion of the Internet, and by trading on people's increasing willingness to purchase goods and services remotely, this company provides what it calls a "sockscription" service, whereby users register their preferences and every three months receive a supply of new socks through the mail. As the jury of the 2001 Swiss Web Awards noted, this service means that consumers can avoid "the tiresome purchase of socks;" thus, the apparently absurd pairing of socks with e-commerce is "turning out to be a clever idea: The market is large, the goods uncomplicated, the dispatch easy." And importantly, for a company that wishes to constantly learn from its consumers and refine its service, "[m]ail subscription affords regular customer contact." In this way, Blacksocks ${ }^{\mathrm{TM}}$ has taken the humble sock and designed a system around it upon which the firm can capitalize.

Throughout the history of its use, the sock has changed little as a product. At its most basic, as a textile membrane that covers the foot, keeps it warm, and absorbs sweat, the sock is difficult to better. Still, the nature of the material from which socks are made has changed: Cotton came to supersede wool and silk in the nineteenth century; nylon became available in the 1940s; and since then, a range of synthetic fibers, including polyester and Spandex, has been used in their manufacture. Similarly, the mechanics of production have developed over time. The invention of the knitting machine in the sixteenth century meant that socks could be produced more quickly than had been done by hand; the introduction of the circular loom in the 1930s allowed socks to be produced without seams. Despite these innovations, the essential form of the sock as a simple technology of foot covering has remained unaltered. Even the introduction of smart-socks or spray-ons would not be likely to change this in any fundamental way.

Arguably, this stability is due to the very basic nature of the sock as a device and its essential relationship to an enduring need: The shape of people's feet has not changed; the nature of walking has not changed; the requirement for a membrane between the foot and the outer footwear has not changed. Thus, the essential need, in terms of the function to be provided by the product, has remained the same. What can be seen to be changing are the conditions of its production and consumption.

Throughout the latter part of the twentieth century and into the twenty-first, mass-production, the practical and ideological driver of modernity, has moved in its center of gravity from the developed West to the emergent economies in the rest of the Sus for Old Europe: Product Services, Sustainability and Competitiveness (Sheffield, UK: Greenleaf Publishing Ltd., 2006). 
9 Matthew B. Cook, Tracy Bhamra and Mark Lemon, "The Transfer and Application of Product Service Systems: from Academia to UK Manufacturing Firms" The Journal of Cleaner Production 14, no. 17 (2006): 1460.

10 Cook, Bhamra and Lemon "The Transfer and Application of Product Service Systems: from Academia to UK Manufacturing Firms;" Manzini and Vezzoli, Product Service Systems and Sustainability: Opportunities for Sustainable Solutions; Tukker and Tischner, New Business for Old Europe: Product Services, Sustainability and Competitiveness.

11 For customers in Switzerland, Blacksocks ${ }^{\mathrm{TM}}$ provides just such a function, in that each delivery of socks includes a freepost envelope into which the customer puts old or worn out socks to return to a recycling service.

12 Oksana Mont, Functional Thinking-The Role of Functional Sales and Product Service Systems for a Function-based Society, IIIEE (Lund, 2002).

13 See, e.g., Victor Papanek, Design for the Real World (London: Granada, 1978); Tony Fry, Design Futuring: Sustainability, Ethics and New Practice (London: Berg, 2008). world. ${ }^{8}$ The massively reduced labor costs involved have meant that the West could no longer compete on the basis of scale. Consequently, many producers have started to conclude that their future lies not in being the mass manufacturers of objects-traditional material commodities-but as the providers of services and "added value." The development of the discourse of the PSS thus represents a shift from attempts to compete by innovating on economies of scale (which conditions have rendered unavailable) to attempts to develop economies of scope by expanding into the areas in which a business can operate to add, and therefore extract, value. ${ }^{9}$ In this way, Blacksocks ${ }^{\mathrm{TM}}$ —as an example of a company innovating in the field of socks-has found its advantage not in developing a new form of foot covering or discovering a new way of making such things more cheaply, but in changing the way users relate to what they consume. The company is trading on a changed relationship between the consumer and the product's delivery, an altered worldview that sees material things as being implicit in the dynamic structures that allow them to arrive in daily life.

It could then be suggested, as many commentators do, that such a systematization of the process of production and consumption does not benefit just the makers and users of things in such circumstances, but might actually make such commercial practices more sustainable and thus be good for society as a whole. ${ }^{10}$ In business terms, such sustainability emerges in that, as the potential scope expands, companies can keep on trading even when massmanufacturing advantages are removed. At the same time, systems potentially become environmentally more sustainable because the integrated nature of such methodologies allows for practices such as "closed loop" recycling."

Janet's and Faisal's systems could be seen as an enhancement of this approach, whereby both the provider and the wider society benefit, resulting in a "win-win" situation..$^{12}$ Janet's socks are super-durable, they will not wear out, and their morphic qualities mean they are less likely to be replaced with a more fashionable version; meanwhile, Faisal's foot-coverings are ephemeral, in that they wear out quickly, but the recycling process is so immanent to their use that the resources necessary to provide functionality are greatly reduced..$^{13}$ So, such high-tech sock-service systems thus can be seen to de-materialize the provision of socks, and apparently, everybody wins. Yet, if these types of innovations do indeed represent the exploitation of an alteration in the relationship between the consumer and that which is consumed, the question to consider is how anything that can be called "the product" is manifest in a situation that affords such opportunities. 


\section{Locating the Product}

Despite the comments of the award jury, it is unlikely that many people regard the purchase of socks in itself as "tiresome." What can be irritating and perceived as a lack or a need, however, is running out of them. This is the problem that Blacksocks ${ }^{\mathrm{TM}}$ solves: It offers a constant supply of socks without having to think about how or when or where to get new ones. In the process, the intimate relationship developed with the consumer allows the company to access immediate data concerning the needs of its clients, and over time a model of the behavior of this segment of the sock-consuming market can be built and used to further redesign the service. In this way, the actual product being bought has changed: No longer are simply the socks themselves purchased; rather, a whole socksystem has been constructed that is consumed as a form of service.

Services tend to be seen as immaterial. As Lucy Kimbell notes, they are typically conceived in terms of "what products are not"- as being intangible, in opposition to the material tangibility of physical things. They are often conceptualized as having no real physical form but instead are recognized as being "distributed in space and time."14 They are seen as a collection of what Nicola Morelli calls "intangible functionalities" that serve a particular end. ${ }^{15}$ Services are thus characterized as essentially distributed entities that serve to facilitate certain goals.

The development of PSS, as part of what Kimbell describes as "the turn to service design," 16 could be seen as a process by which the service, rather than the material artifact, is becoming the entity consumed; consequently, service can be understood to be the subject of the process of design, manufacture, and marketing, with the shaping of physical objects being only part of this much larger scope of activity. This shift has arguably been intensified in recent years with the development of a more networked way of living, one predicated upon the rise of new communication and interaction technologies that have often served to make the things we buy and use less discretely material. In such a situation, changes in the wider social and cultural context-in which the business of making, selling, and using takes place-mean that new forms of product are actually being created. These forms appear less tangible because they are more distributed in time and space than the material products they have come to absorb-products that had uncomplicatedly been identified as this or that individual artifact. As a result, the provision of services appears to be an ideal way to dematerialize provision, in that the reduction of the physical resources necessary to achieve the desired ends seems clearly to have the potential to make such processes more ecologically sustainable, even as they make business more profitable. Exploration" Design Issues 18, no.3 (2002): 5

16 Lucy Kimbell "The Turn to Service Design," 157. 
In terms of their mereological status, services, from the perspective of use, can be understood as a form, or type, of product. The service is what the purchaser buys-what the customer receives for their outlay in the pursuit of certain ends. Thus, the service is recognizably a product that the user consumes; the service can be observed to be part of the category "product." Yet suggesting that such a situation unproblematically means that the service becomes the entirety of the product would be a mistake. What has changed is that the product has ceased to be completely mapped onto the physical object and comes to be distributed in both time and space. In the process, it has come to flow into the practices of daily life. In such a model, the product-what is being made, sold, and consumed-can be seen to be meshing into the structures it inhabits. It is no less a product as a result; instead, it has changed its nature. The product as an entity has come to encompass the material artifacts created, the service provided, and the conditions in which the two are consumed.

Although such products are distributed into the apparatus they inhabit, they are not, as Morelli has claimed "processes which exist in time only."17 They may have become less discretely material as products, but they do still definitely exist in space as entities with physical components. ${ }^{18}$ The material conditions necessary for their existence and operation have not evaporated or, in many cases, even been reduced. Instead, diffuse products have been developed that have their parts woven into the fabric of consumption as a process.

These changes represent a shift in the conditions of design. Here, the designer must still be able to effect the formation of artifacts (the constitution of Janet's super-durable textile or Faisal's recycling unit are properly engineering problems), as well as the processes that bring them into being. But the more knotty question is exactly how the product, as distributed into lived human behavior, is to be designed. The proposition here, then, is that the answer depends on why (i.e., to what ends) such design is being undertaken.

The designer thus becomes the one who gives form to what Systems: A Methodological Exploration," 5 .

18 For an examination of the different perspectives that might be adopted with regard to the materiality of services, see Lucy Kimbell, “Designing for Service as One Way of Designing Services," International Journal of Design 5, no. 2 (2011): 41-52.

19 See particularly Liz Moor and Guy Julier "Introduction: Design and Creativity" in Liz Moor and Guy Julier, eds., Design and Creativity: Policy, Management and Practice (Oxford: Berg, 2009), 1-22. 
20 See Jane Fulton-Suri, "The Experience of Evolution: Developments in Design Practice," The Design Journa/ 6, no. 2 (2003): 39-48; Elizabeth Shove, Matthew Watson, Martin Hand, and Jack Ingram, The Design of Everyday Life (Oxford: Berg, 2007).

21 B. Joseph Pine and James Gilmore, The Experience Economy (Boston: Harvard Business School Press, 1999); Jonathan Cagan and Craig Vogel, Creating Breakthrough Products: Innovation from Product Planning to Program Approval (New York: FT Press, 2002).

22 For an outline of such approaches, see Pieter Desmet and Paul Hekkert, "Framework of Product Experience" International Journal of Design 1 , no. 1 (2007): 57-66; Deana McDonagh, Paul Hekkert, Jeroen Van Erp and Diane Gyi, eds., Design and Emotion: The Experience of Everyday Things (London: CRC Press, 2003).

23 Julka Almquist and Julia Lupton, "Affording Meaning: Design-Oriented Research from the Humanities and Social Sciences," Design Issues 26 , no. 1 (2010): 3.

24 Donella Meadows Thinking in Systems: A Primer (Vermont: Chelsea Green, 2008).

\section{Designing Wearing}

The planning and implementation of a large-scale sock-system is clearly a bigger problem than designing an individual pair of socks. Because of the intimate relationship that must be developed between the producer and the consumer, such an undertaking necessarily involves a more detailed knowledge of the nature of use. The development of the idea that the perceived value of what is made, sold, and used can be improved through the study of the practices of consumption-what has come to be termed "user-centered design" - has led to a fundamental shift in the perceived nature of design and the role of the designer. ${ }^{20}$ As a result, the scope of design as a practice has been reconfigured, moving from the shaping of artifacts to the forming of user experiences. ${ }^{21}$ Consequently, the designer is increasingly expected to have a range of competencies that relate to understanding the behavior of consumers and their cognitive processes. To facilitate such understanding, a whole battery of methodologies has been developed in the name of design research, from qualitative ethnographic interviews and cultural probes, to more clinical and behaviorist analyses of specific interfaces and product experiences..$^{22}$ Such studies, which are intended to reveal the nature of use, have, as Julka Almquist and Julia Lupton put it, remapped design research from the study of things to the study of people. ${ }^{23}$ The perceived subject of designwhat is to be designed-is no longer the material artifact in isolation but the actual conditions of its use. This means that to design use is inevitably to be involved, to some extent, in the design of human subjectivity itself.

The way in which the "user" is imagined is crucial because the nature of any system, its character, initially depends on its ontological schema-on the entities that are deemed to exist as part of it. Such a structure is established according to the specified ends or purpose of the system. ${ }^{24}$ For example, in any area of the world, if the correct data about sock consumption were available, such use could be mapped: Energy input and the efficiency of provision could be observed; the behavior of the users could be codified and algorithms constructed to model such practices and shape the activities of the consumers. In this way, an organization with the necessary infrastructure could supply socks to people as a population. The most efficient way to do so might not then be Janet's highly personalized system, or even Faisal's individual spray-on set-up, but the use of a centralized grid or network of facilities. Here, each building or city block might have a sock-station where consumers would queue-up each morning to have their socks applied. In such a scenario, individual choice would inevitably need to be curtailed to some degree; we can easily imagine, for example, that grey socks for all might be the most resource-efficient solution. 
25 Oksana Mont, Functional Thinking-The Role of Functional Sales and Product Service Systems for a Function-Based Society, 3.

26 Ibid, 19

27 The technological equipment of daily life can be understood as a form of material ideology, which is the physical mechanism that iterates, actualizes, and perpetuates power structures. As Althusser states in his analysis of the action of ideological reproduction of the conditions of production, "an ideology always exists in an apparatus, and its practice or practices. This existence is material." That is to say, through our experience of the material world, we are constituted as particular subjectivities. Louis Althusser, Essays on Ideology (London: Verso, 1993 [1970]), 40.
Such a model of standardized provision would almost certainly result in a more rationalized use of resources than one that allows for an individualized form of consumption. According to Oksana Mont, for this kind of systemic approach to work at maximum efficiency, and thus be sustainable in the long run, a radically functionalist approach has to be adopted, whereby producers become "function providers" and a "function-based society," as she calls it, is established. ${ }^{25}$ In this vision, what is sold to the consumer as a product is function itself, rather than the means to achieve it. So, rather than selling energy, gas, or electricity, which a consumer could use to heat water, the producer offers a hot water service; or rather than simply selling socks, the functionalities of them-such as having warm, covered feet, etc.- -are made available; and in such a situation, Mont argues, "technologies can be considered to be mere modes of providing function." ${ }^{26}$ The problem with such an approach is that no technology or material apparatus can ever be a "mere" mode of provision for some ideal and unchanging function, abstracted from the form of daily life in which it is embedded.

Technology is the apparatus of life; ${ }^{27}$ it is the material substrate from which social action is formed. It always operates performatively, in that what we live with, structures how we live. Just as the dematerialization of the product is not its immanent immateriality but its distribution into the apparatus, so the qualities of the delivery mechanism are both formed by, and serve to form, the epistemological and ontological possibilities of the system.

Both an individual pair of socks and a complex integrated municipal sock-provision system are forms of technology; they are each in themselves mechanisms that provide users with the capacity to fulfill certain functions. In these alternate kinds of provision, the objective of the system is constituted by its posited teleological end-point-its final cause or purpose-which then establishes the nature of the interrelationships of the parts. The way in which the parts interact in the intended provision of the outcome then forms the character of the system. However, because use is part of the system in the service-system paradigm, with each mechanism different human subjects are not simply catered to, but generated. Thus, the product designer becomes a population designer-this is then a situation that should make us more than a little uncomfortable.

\section{The Why of Systems}

Given our current state of technological development, a mass sock-provision system like the one described above could be implemented, but what would it mean for somebody to actually live like this? Given the rise of the PSS paradigm, this then becomes a question the designer must consider. This is because it represents a 
shift from design being primarily a practice concerned with questions of extensive materialism (i.e., the nature of the form and function of this or that object as a physical element in a process) to one that must operate in the field of intensive dynamics, as the nature of the functioning of the whole, the entirety of the process including its ultimate effects, comes to be the subject of design, the fundamental problem to be addressed. Thus, to understand products as distributed beyond the individual material artifact is to see design as a practice involved with the conceptualization of the wider structure in which the product operates, and which in the process it perpetuates.

In the systemic paradigm, design can be seen to be motive, in that it is concerned not only with motion and the dynamics of how things work, but also their purpose-the why of the system. As a practice, design is essentially involved in the identification of what needs to be done, the establishment of a means for achieving this, and the nature of the intended outcome-whether this projected result is the making of a nozzle or the engineering of a population. So, the problem-setting that is implicit to design work is concerned with the establishment of what needs to be done, what Aristotle called first cause, and the relationship of this initial point of action to the teleological end point, or final cause, of the process ${ }^{28}$ How the designer acts to specify such positions is then an ethical question, in that it is concerned not just with finding and solving problems, but with their definition. Such definitions then determine what the effective response will be, the material nature of the solution, and the form the results can take.

Technological capacity cannot set the limits of the possible because the structure of the equipment in operation is already the expression of a particular world-view. A study of consumers and their needs cannot set the criteria because the agency of these actors will be constructed in the process. Instead, the application of a set of values must (and will) define the purpose of any designed response to a problem. As Alain Findeli has observed, in design a solution can never be true or false in absolute terms; rather, it can only be more or less appropriate. ${ }^{29}$ Thus, to identify the "why" of the system is to make an ethical judgment about the nature of the outcome. In establishing the problem to be solved, the designer must address the question of what the effects of this intervention will be-not what the intended outcome is, but what the design has the potential to do.

description of Aristotle's conception of ethics and causality in "Intensive Culture," (2006), a paper delivered at the Centre for Research Architecture, Goldsmiths College, University of London, http://roundtable.kein.org/node/414 (accessed December 12, 2010).

29 Alain A. Findeli, "Ethics, Aesthetics and Design," Design Issues 10, no. 2 (1994): 61. 
use shopping as an active and constructive experience. Comparison shopping; hunting for bargains; the choices of where, when, how and why to go shopping are all bounded points of negotiation, but they do allow for a certain constitution of the subject as an acting agent. ${ }^{30}$ Now, it seems, shopping is "tiresome." So the system will even do this task for you, with the promise that it will simplify your life.

However, as consumption has become more digitally dependent, the systems we use to obtain what we need have become more distributed, more complicated, and more difficult to comprehend. As a result, human actors have, to an extent, effectively contracted out their agency to the structures upon which they depend. In the late 1990s, Nicholas Negroponte talked glowingly about how "digital agents" would emerge as the dominant form of humancomputer interaction. He compared them to travel agents or real estate agents, who combine a knowledge of their subject with the ability to model users and assess their needs. ${ }^{31}$ This approach is no longer simply possible; it has now become an intrinsic part of the experience of consuming systems.

Throughout the twentieth century, the trend was toward the consolidation of the consumer experience. The development of department stores meant that many needs could be satisfied from different parts of the establishment with only a single payment; malls enabled consumers to do all the shopping they needed while finding only one parking space; Amazon and eBay have created one-stop locations on the web for exercising a degree of the choice that is held sacrosanct as a measure of autonomy in consumer culture. The introduction of the PSS model actually has the potential to reverse this trend, and not in a good way. It can create the conditions whereby, even as provision becomes more integrated, the consumer experience becomes more fragmented and the individual has less control. As the initiative in consumption and research shifts from the self to the system, the methods that emerge from such an approach inevitably separate the consumer, to one degree or another, from the ongoing need to make choices, to exercise agency, in the act of consumption. That is to say, the self authorizes the system to act on its behalf, and in doing so, gives up a certain degree of autonomy. The deal then depends on the promise that this approach will make life easier.

Consuming a system rather than purchasing individual components and acting to combine them to affect the necessary function might have certain practical implications that produce unforeseen complications for end users. For example, unless all of their needs are met by one all-encompassing system, they will

30 Daniel D. Miller, A Theory of Shopping (New York: Cornell University Press, 1998).

31 Nicholas Negroponte, Being Digital (London: Coronet, 1996), 154-56. 
situation, the consumer will then have a new role, as the one who acts to ensure the integration of these elements into their life. They are incited to become pseudo-employees of the companies involved, tasked with connecting the elements of complex processes. In this way, the user comes to be distributed into the systems they consume, as the actual experience of consumption becomes fragmented.

\section{Conclusion}

To design a system in which people spray on their socks in the morning is to propose that such a scenario offers an acceptable way to live. By becoming more than the shaper of an individual material artifact and envisioning and constructing systems of provision, the designer necessarily takes on a more explicitly ethical role. Such a position operates at the level of problem setting, of identifying the product and the telos-or final cause-of the process, of establishing the "why" of the system. Such justifications will then depend upon certain values that come to act protologically in the action of the system's operation.

Both Janet's luxury service and Faisal's utilitarian kit are extreme examples of an over-engineered solution to a problem. We do not need to love socks; we just need them to work. We should not be recycling socks; they should just last a long time. Why do people need to keep on buying so many socks? Given the technological capabilities available, the creation of socks that do not wear out would not seem to be beyond our collective productive capacities. Indeed, as was observed at the beginning of this article, they already exist, but the space to make this choice has not been opened up. They are not made readily available because it is not profitable to do so.

Producers want to become service providers not because doing so results in a "win-win" situation for everybody; they want to do it so they can stay in business, even though their previous field of operation has shifted from under them. For all of the proposed potential for approaches such as PSS to provide a more sustainable future in ecological terms, it seems clear such concerns are not presently the main driver of changes in practice. ${ }^{32}$ The development of products that are distributed into the apparatus of social life are primarily being designed in this way to make business more sustainable-to perpetuate a certain mode of production in its present form. On the level of consumption, the goal is to absorb as much of the user's agency as possible into the process to make provision more efficient and profitable, even as doing so often occurs at the expense of making the lives of consumers more,

32 Cook, Bhamra and Lemon, "The Transfer not less, complicated. 
The development of a systemic paradigm - to see making and use, production and consumption as part of the same process in which all actors have a stake-does offer real potential for change. The main challenge that designers face in such a situation is to recognize that the practice of design is necessarily concerned with ethics. Not simply because the designer has to consider what is "right" in any circumstance, but because they must first be able to identify the problem to be solved. The nature of this problem then turns on why it is to be addressed at all, and whose interests, in the end, the solution is to serve.

\section{Acknowledgement}

This research is part of the Creative Industry Scientific Program (CRISP), which focuses on the design of product-service systems as a means to stimulate the continuing growth of the Dutch Design Sector and Creative Industries. The CRISP program is partially sponsored by the Dutch Ministry of Education, Culture and Science. 